\title{
State-Dependent Perceptual Learning
}

\author{
Frank Freyer, ${ }^{1,2,3}$ Robert Becker, ${ }^{1,2}$ Hubert R. Dinse, ${ }^{3 *}$ and Petra Ritter ${ }^{1,2,4,5 *}$ \\ ${ }^{1}$ Bernstein Focus State Dependencies of Learning and Bernstein Center for Computational Neuroscience, Berlin, Germany, ${ }^{2}$ Department Neurology, \\ Charité-University Medicine, Berlin, Germany, ${ }^{3}$ Institute for Neuroinformatics, Neural Plasticity Laboratory, Ruhr-University Bochum, Germany, ${ }^{4}$ Max \\ Planck Institute for Human Cognitive and Brain Sciences, Leipzig, Germany, and ${ }^{5}$ Berlin School of Mind and Brain and Mind and Brain Institute, Humboldt \\ University, Berlin, Germany
}

Learning constitutes a fundamental property of the human brain-yet an unresolved puzzle is the profound variability of the learning success between individuals. Here we highlight the relevance of individual ongoing brain states as sources of the learning variability in exposure-based somatosensory perceptual learning. Electroencephalogram recordings of ongoing rhythmic brain activity before and during learning revealed that prelearning parietal alpha oscillations as well as during-learning stimulus-induced contralateral central alpha changes are predictive for the learning outcome. These two distinct alpha rhythm sources predicted up to $64 \%$ of the observed learning variability, one source representing an idling state with posteroparietal focus and a potential link to the default mode network, the other representing the sensorimotor mu rhythm, whose desynchronization is indicative for the degree of engagement of sensorimotor neuronal populations during application of the learning stimuli. Unspecific effects due to global shifts of attention or vigilance do not explain our observations. Our study thus suggests a brain state-dependency of perceptual learning success in humans opening new avenues for supportive learning tools in the clinical and educational realms.

\section{Introduction}

Perceptual learning describes the ability to improve perception as a function of repeated sensory experience through training, practicing, or mere passive sensory stimulation (Fahle and Poggio, 2002; Seitz and Dinse, 2007; Sasaki et al., 2010). It is, however, a common observation that despite intact sensory and cognitive functions of the participants, the learning rate varies greatly across subjects, and subpopulations of subjects often show little or no learning (Fahle et al., 1995; Fahle and Henke-Fahle, 1996; Hodzic et al., 2004). While there is agreement that attention is a key factor that can determine the perceptual learning outcome (Crist et al., 2001), examples exist where perceptual learning can be induced without attention, e.g., by task-irrelevant (i.e., unattended) or subthreshold stimuli (Watanabe et al., 2001). Therefore other factors might impose additional constraints on the learning process and might also determine the intersubject variability. Recently, evidence has been provided that the large-scale resting-state network activity of the brain (Raichle, 2006) has a considerable impact on perceptual performance (Sigman et al.,

\footnotetext{
Received Aug. 23, 2012; revised Nov. 22, 2012; accepted Dec. 14, 2012.

Author contributions: F.F., H.R.D., and P.R. designed research; F.F. performed research; F.F. analyzed data; F.F., R.B., H.R.D., and P.R. wrote the paper.

We acknowledge the support of the German Ministry of Education and Research (Bernstein Focus State Dependencies of Learning 01GQ0971) to H.R.D. and P.R., the Deutsche Forschungsgemeinschaft—DFG (SFB 874) to H.R.D., the James S. McDonnel Foundation (Brain Network Recovery Group JSMF22002082), and the Max-Planck Society (Minerva Program) to P.R. We thank A. R. McIntosh for his helpful input.

${ }^{*}$ H.R.D. and P.R. shared senior authorship.

Correspondence should be addressed either of the following: Hubert R. Dinse, Institut fur Neuroinformatik, Ruhr University, Building NB 3, 44780 Bochum, Germany, E-mail: hubert.dinse@rub.de; or to Petra Ritter, Max Planck Institute for Human Cognitive and Brain Sciences Leipzig and Department of Neurology, Charité Universitaetsmedizin, Charitéplatz 1, 10117 Berlin, Germany, E-mail: petra.ritter@charite.de.

DOI:10.1523/JNEUROSCI.4039-12.2013

Copyright $\odot 2013$ the authors $\quad 0270-6474 / 13 / 332900-08 \$ 15.00 / 0$
}

2005; Lewis et al., 2009; Baldassarre et al., 2012). Closely related to resting-state network activity are spontaneous ongoing neuronal dynamics, which play a critical role in neural processing of sensory information (Raichle, 2006; Deco et al., 2011), and serve as a marker of an individual's brain state. For example, oscillatory ongoing activity has been shown to be closely related to perceptual performance (Linkenkaer-Hansen et al., 2004; Boly et al., 2007; Hesselmann et al., 2008; Schubert et al., 2009), memory processing (Sauseng et al., 2009), and motor behavior (Mazaheri et al., 2009). Moreover, imaging studies in humans and animal models have shown that trial-to-trial variability in evoked responses, a universal feature of stimulus processing, can largely be explained by fluctuations of the underlying neuronal background activity (Boly et al., 2007; Fox et al., 2007; Becker et al., 2008a, 2011; Mazaheri and Jensen, 2008). Because oscillatory brain states exhibit unique patterns of permanent fluctuations that are different across individuals, one might expect that learning variability could be, to some extent, also related to local ongoing neuronal oscillatory activity, reflecting the individual's brain state that the learning stimulus is encountering. It is therefore conceivable that oscillatory brain rhythms also influence perceptual learning. If this is true, different manifestations of ongoing oscillations reflecting different brain states that either facilitate or hinder perceptual learning, should have a major influence on perceptual learning outcome. To test this hypothesis, we investigated the relationship between perceptual learning and ongoing neuronal oscillatory activity as recorded by electroencephalography (EEG).

\section{Materials and Methods}

Subjects and experimental protocol

Twenty-six healthy, right-handed subjects ( 3 male, $26.1 \pm 4.0$ years) participated and gave written informed consent before the study, which 
A
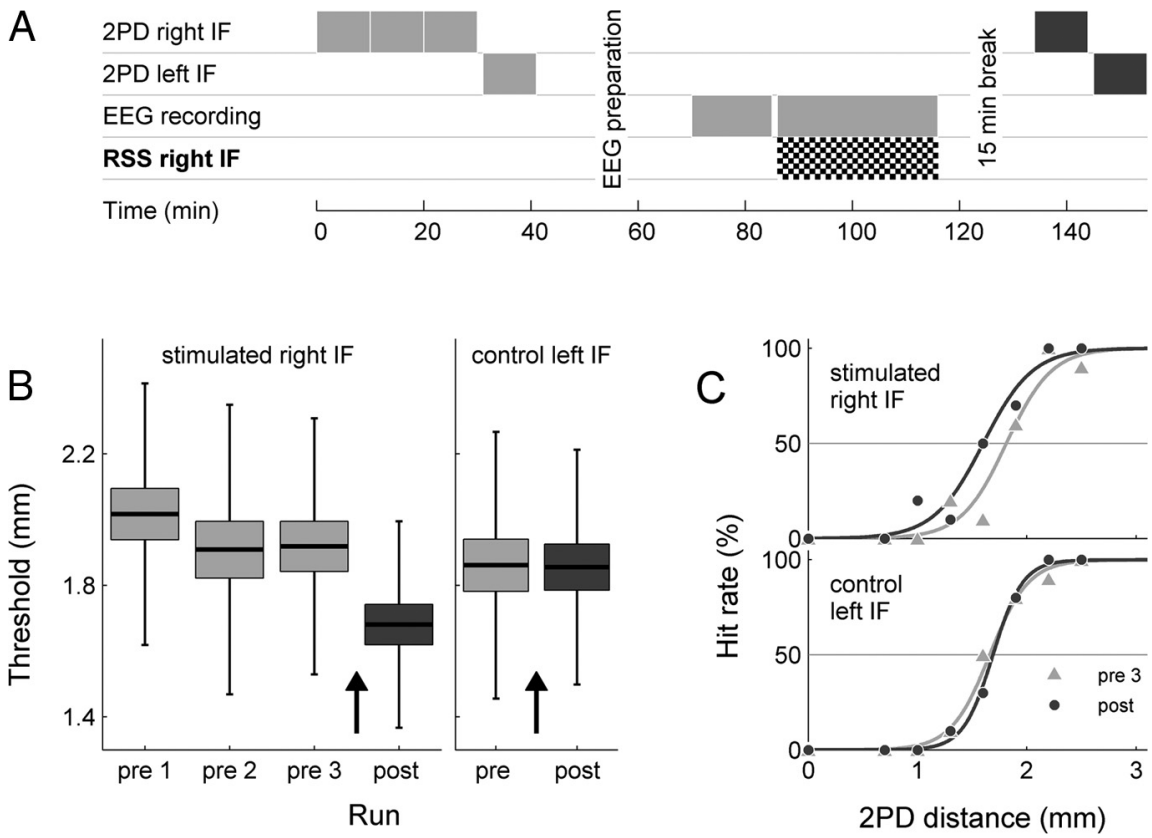

Figure 1. Experimental layout and behavioral results. $A$, Experimental protocol. $B$, Group $(n=26)$ mean 2PD detection threshold (black bar), SEM (box), and SDs (whiskers) for pre (light gray) and post (dark gray) runs. RSS (black arrows) leads to a significant decrease in discrimination thresholds $\left(p<4.67 \times 10^{-6}\right)$ for the stimulated right IF, but not for control left IF ( $p<$ 0.906). For the right IF, pre-RSS results are shown for three consecutive runs, which were performed for baseline stabilization. $C$, Detection rates for the eight different distances and the fits to the psychometric functions for runs pre 1 (light gray, triangles) and post (dark gray, circles), for right stimulated and left control IF of a representative subject. The detection threshold was determined as the distance for which a level of $50 \%$ correct responses was reached.

was performed in compliance with the relevant laws and institutional guidelines and approved by the ethics committee of the Charite University Berlin. Figure $1 A$ shows the timeline of the experimental protocol. In the pre-session, subjects conducted three two-point discrimination (2PD) task runs with the right index finger (IF), to obtain a stable discrimination-performance baseline. Only the third run was used as $2 \mathrm{PD}$ baseline for further analyses. A fourth $2 \mathrm{PD}$ task run was performed with the left unstimulated IF, which we used as a control condition. Subsequently, 15 min of resting state EEG was recorded. In the repetitive sensory stimulation (RSS) session that followed, trains of somatosensory stimuli were delivered for $30 \mathrm{~min}$ to the right IF. EEG was acquired during the whole session. In the final post session, 15 min after termination of the RSS, subjects performed one 2PD task run for each IF.

\section{High-frequency RSS}

We used electrical and cutaneous stimuli for RSS. Both techniques had been shown to induce equivalent improvement of tactile acuity implying that the amount of stimulation-induced improvement of tactile discrimination is not dependent on the type of either cutaneous or electrical stimulation. In 20 subjects, electrical stimulation was applied using two adhesive surface electrodes with an area of $15 \times 20 \mathrm{~mm}$. These were attached to the palmar skin of the right IF, with the positive electrode applied to the distal phalanxes and the negative electrode applied to the proximal phalanxes. In 6 subjects, cutaneous stimulation was applied using a small loudspeaker diaphragm with a diameter of $\sim 8 \mathrm{~mm}$, mounted to the tip of the right IF, and was used to transmit the tactile stimuli of the RSS protocol to the skin (Godde et al., 2000; Pleger et al., 2001; Dinse et al., 2003). Stimulation trains of $1 \mathrm{~s}$ in duration, consisted of 20 single pulses applied at $20 \mathrm{~Hz}$ with an intertrain interval of $5 \mathrm{~s}$. Total duration of the RSS protocol was $30 \mathrm{~min}$, resulting in a total number of 6000 pulses. Intensity of electrical stimulation was set to $50 \%$ above sensory threshold, cutaneous stimulation was suprathreshold, and peakto-peak amplitude of the loudspeaker diaphragm was $\sim 100 \mu \mathrm{m}$ according to laser vibrometer measurements.
Psychophysical measurements

Tactile discrimination was assessed using the method of constant stimuli by measuring simultaneous spatial 2PD thresholds (Godde et al., 2000; Pleger et al., 2001; Dinse et al., 2003): subjects were tested using $0.7,1.0,1.3,1.6,1.9$, 2.2 , and $2.5 \mathrm{~mm}$ separation distances. In addition, zero distance was tested with a single needle as a control for false alarms. The needles were mounted on a rotatable disk that allowed switching rapidly between distances. To accomplish a uniform and standardized type of stimulation, the disk was installed in front of a plate that was movable up and down. The arm and fingers of the subjects were fixed to the plate, which was moved up and down by the experimenter. The down movement was halted by a stopper at a fixed position above the needles. The test finger was held in a hollow containing a small hole through which the finger came to touch the needles, at approximately the same indentations in each trial. Each distance, including zero distance, was tested 10 times in pseudo-randomized order, resulting in 80 single trials per session $(\sim 10 \mathrm{~min})$. The subjects had to decide immediately if they had the sensation of one or two tips by answering "one" or "two."

For each session, subjects' responses to the $2 \mathrm{PD}$ task were summed and plotted against the distance as a psychometric function for absolute perceptual threshold. Psychometric functions were fitted using the psignifit toolbox, version 2.5.6 for Matlab (see http://bootstrap-software.org/psignifit/), which implements the maximum-likelihood method described by Wichmann and Hill (2001). Based on the fitted curve, the perceptual threshold was defined as the distance for which $50 \%$ correct responses were obtained. The first two $2 \mathrm{PD}$ runs in the pre-session were conducted to achieve a stable baseline before RSS. Only the third run was used for further analysis.

The percentage threshold change of the 2PD task between pre- and post-sessions served as an index of perceptual learning outcome. A decrease of the threshold was taken as an indication of an improvement in tactile acuity. To be classified as a "learner," the subject's threshold decrease of the right stimulated IF had to be greater than the $95 \%$ confidence interval of the mean threshold change in the left (control) IF. Using this criterion, each subject was classified as either "learner" or "no-learner."

For both hands, grand average threshold changes (pre- vs postsession) were tested for significance using a paired Student's $t$ test (normality of the distribution was confirmed by an Anderson-Darling test).

\section{EEG recording settings}

Scalp EEG was recorded using a 64 channel-EEG system (BrainAmp, Brain Products $\mathrm{GmbH}, 0.1-250 \mathrm{~Hz}$ hardware bandpass filter, 61 scalp channels arranged according to the International 10-20 System, two electrocardiography channels, and one vertical EOG channel, all referenced against $\mathrm{CPz}$, impedances $<5 \mathrm{k} \Omega$ ). During all EEG recordings, subjects sat in a quiet and dimly lit room and were instructed to stay awake and watch a silent animal documentary on a computer screen (at a distance of three meters distance). This allowed maintenance of a high state of vigilance, while minimizing eye movements.

\section{Data analysis}

Psychophysical measurements. Subjects' 2PD responses were summed and plotted against the distance as a psychometric function. Threshold was defined as the distance for which $50 \%$ correct responses were obtained. Grand average threshold changes (pre- vs post-session) were tested for significance using a paired Student's $t$ test. 
EEG. EEG data analysis was performed using MATLAB and the EEGLAB toolbox (Delorme and Makeig, 2004). EEG was down-sampled to $100 \mathrm{~Hz}$ and filtered $(1-40 \mathrm{~Hz})$ to remove slow drifts and line-noise, and to improve the frequency specificity of subsequent postprocessing techniques. EEG data were visually inspected and segments of gross movement artifacts (due to movements or bad electrode impedances) were excluded. In all datasets, such segments constituted $<2 \%$ of the data, indicating an overall sufficient data quality. For each subject, EEG datasets of all three sessions (pre, RSS, post) were merged and EEG scalp channel data (61 channels) were submitted to an extended Infomax independent component analysis (ICA). ICA linearly unmixes the original EEG channel data into a sum of maximally temporally independent and spatially fixed components (Bell and Sejnowski, 1995). For each of the resulting independent components (ICs) we calculated power spectra using Fast Fourier transforms (FFTs, resolution $0.5 \mathrm{~Hz}$ ), somatosensory evoked potentials (SEPs) timelocked to the sensory stimulus, and eventrelated spectral perturbation (ERSP) images (see EEG- event-spectral perturbation).

Visual inspection of time courses, spectra, and topographic distributions was used to identify ICs that reflected eye movement, scalp muscle artifacts and movement artifacts. These ICs were removed from the data. To ensure that the RSS stimuli were correctly applied and adequately processed cortically, we scanned all remaining ICs in each subject for components representing activity related to the somatosensory processing of the stimulus (henceforth referred to as $\mu \mathrm{IC}$ ). To be identified as $\mu \mathrm{IC}$, four criteria had to be met: (1) a clear sensorimotor topography of the component map, (2) a clear alpha $(8-12 \mathrm{~Hz})$ frequency peak in the component spectrum (a beta peak is usually expected, but is not obligatory), (3) an SEP with a significant primary amplitude response (P50), and (4) a significant event-related desynchronization (ERD) in the alpha frequency band for the somatosensory stimuli (Cheyne et al., 2003; Gaetz et al., 2006) applied during the RSS session (see EEG-event-spectral perturbation). Figure 2 shows the properties of a $\mu \mathrm{IC}$ in a representative subject. The component exhibits a clear sensorimotor topography, a spectral peak in the alpha and beta band, and both an evoked and an induced response to the stimulus.

Identifying components extracted by the ICA that were related to somatosensory processing, was primarily to ensure that the RSS was successfully applied and to identify the peak frequency of the central alpha rhythm. However, we performed all subsequent analyses in channel space and not in component space because we did not want to bias our analysis a priori to only ongoing activity related to the sensorimotor system. A considerable fraction of rhythmic brain activity relevant to perceptual learning might be hidden in other components that were not categorized as $\mu \mathrm{ICs}$.

For each subject, the individual peak frequency ( $\mathrm{I} \alpha \mathrm{f})$ of the central alpha rhythm was determined as the frequency in the power spectrum of the $\mu \mathrm{IC}$ showing the maximum value within the broad alpha $(8-12 \mathrm{~Hz})$ band. The following frequency bands were defined: delta $(1-3 \mathrm{~Hz})$, theta (4-7 Hz), alpha (I $\alpha \mathrm{f}-0.5 \mathrm{~Hz}$ to $\mathrm{I} \alpha \mathrm{f}+0.5 \mathrm{~Hz})$, beta $(13-29 \mathrm{~Hz})$, low gamma $(30-40 \mathrm{~Hz})$. We calculated two EEG parameters separately for each subject, channel, session and sub-band: (1) the average spectral power during the resting state pre-session before RSS (Welch's method, Hamming-windowed segments, length 2 s, $50 \%$ overlap, resolution 0.5 $\mathrm{Hz}$ ), and (2) the event-related induced change in spectral power due to the somatosensory stimulus during RSS (see next paragraph).

EEG-event-related desynchronization. We calculated the eventrelated induced change in spectral power due to the somatosensory stimulation, separately for each frequency band. EEG time series data from each channel were segmented into epochs starting $1 \mathrm{~s}$ before and ending
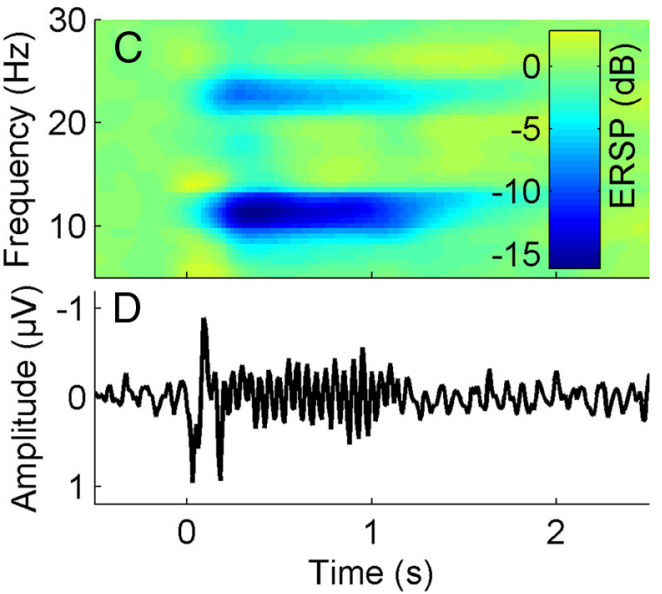

Time (s)

2. Properties of an independent component reflecting $\mu$-rhythm activity $(\mu \mathrm{IC})$ in a representative subject. $\boldsymbol{A}$, Topo政 sustained for one second. Note the pronounced ERD in the alpha and beta frequency band during stimulation. $\boldsymbol{D}$, Corresponding averaged somatosensory evoked potential.

$4 \mathrm{~s}$ after the first pulse of each 20-pulse train, resulting in 300 epochs of $5 \mathrm{~s}$ duration. Each epoch was then transformed to a spectrographic image using a moving-window average of FFT spectra with a resolution of 0.5 $\mathrm{Hz}$. Each spectrographic image corresponds to a data matrix, with rows representing frequencies and columns representing time points. Mean ERSP images were obtained by subtracting the mean log baseline power $(-1 \mathrm{~s}$ to $-0.5 \mathrm{~s})$ from the $\log$ power at each frequency and latency in each trial, and then averaging the resulting images over all trials (see (Delorme and Makeig, 2004) for a detailed description of ERSP calculation). The use of the log power spectrum reveals the relative event-related changes in spectral power, expressed in decibels. Significant intrasubject mean changes in power from baseline $(p<0.01)$ were identified using permutation statistics. Because the time and frequency extension of the ERD can be highly variable across subjects, we did not use a fixed timefrequency window to calculate ERSP. In each subject, we used the ERSP of the $\mu$ IC to identify the largest cluster of significant RSS-induced ER$\mathrm{SPs}$, separately for each investigated frequency band. The time-frequency extension of the respective ERSPs was used as a mask, and frequencyspecific ERSPs were calculated in each channel by taking the mean log power value within this mask.

\section{Correlation analyses}

Pearson's linear correlation coefficients were calculated for psychophysical parameters (2PD changes for stimulated and control IF), and EEG parameters (resting-state power, ERD), across subjects, but separately for each channel. Normality of the data was ensured by the AndersonDarling test.

\section{Correction for multiple comparisons}

Correlation analyses were conducted separately for each channel to yield information about a possible topography. This approach increases the probability of type I errors (false alarms), and thus mandates a correction for multiple statistical testing (or multiple comparison). To this end, we used a cluster-based nonparametric statistical framework (Bullmore et al., 1999; Maris and Oostenveld, 2007), which controls the false alarm rate at a fixed alpha-level, regardless of the underlying statistic. Significant channels $(p<0.01)$ were clustered on the basis of temporal adjacency. The cluster-level statistic was defined as the sum of all $t$-values (or $F$-values) within a cluster. The same procedure was applied to a large number $(N=2000)$ of surrogate datasets, in which the two conditions were randomly shuffled between subjects.

The cluster-level statistic of the largest cluster of each permutation provides a permutation distribution of maximum cluster statistics occurring by chance. A cluster of the true dataset was considered significant if the observed 
Correlation: $\alpha$-band baseline power vs. 2PD change

A

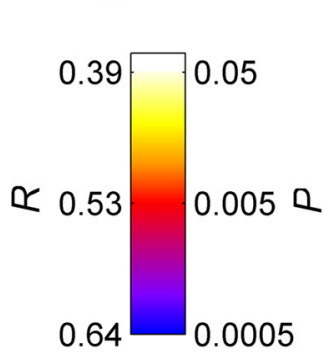

right stimulated IF
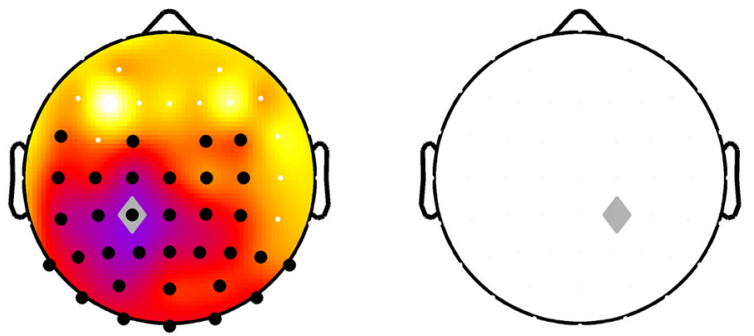

B

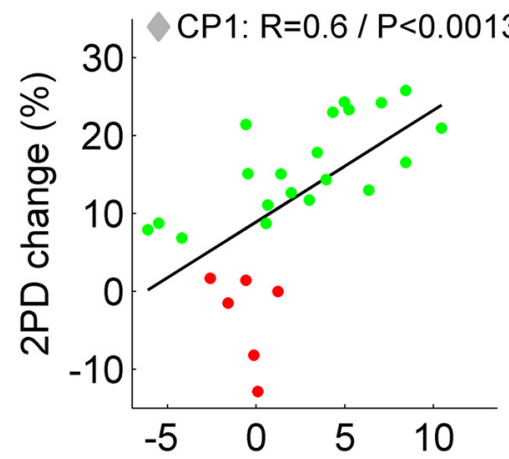

3

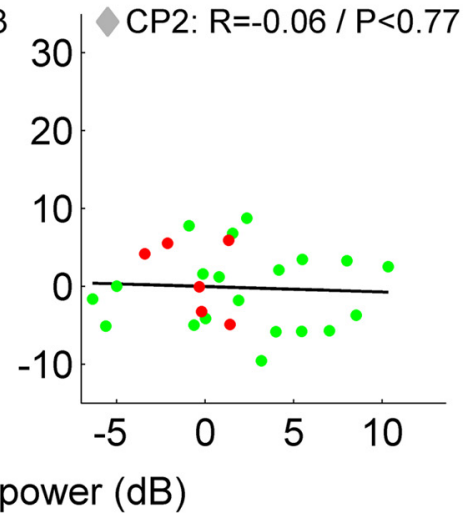

Figure 3. Correlation between $2 \mathrm{PD}$ change (left column: stimulated right IF; right column: control left IF), and alpha band resting-state EEG power before perceptual learning. $A$, Top row, Scalp distribution of Pearson's correlation coefficients $R$ and corresponding $P$-values. Black dots, channels within significant cluster $\left(p_{\text {clust }}<0.002\right)$. Gray diamond, Channel CP1 with maximum correlation to learning rate (for right IF)/corresponding contralateral channel CP2 (for left IF). B, Scatter plot of single subject values at channels (P1/CP2. Successful learners in green $(n=20)$, other subjects in red for illustration purposes-no categorization/collapsing was done for correlation analysis.

cluster statistic exceeded the 97.5 percentile of the permutation distribution (leading to a critical alpha-level of 0.05 of a two-sided test).

\section{Results}

To induce perceptual learning, we took advantage of a learning approach, where perceptual improvement is induced not through training and practicing, but through repeated exposure to high-frequency RSS (Godde et al., 2000; Pleger et al., 2001, 2003; Dinse et al., 2003, 2006; Ragert et al., 2008, Beste et al., 2011). As a psychophysical measure of perceptual learning, we assessed tactile acuity before and after RSS. As a measure of neuronal activity we acquired EEG before and during the perceptual learning process.

Perceptual learning — increased tactile acuity after RSS After three runs of the $2 \mathrm{PD}$ task with the right IF, subjects reached a stable discrimination-performance baseline (ANOVA of the three runs: F: $0.55, p<0.578$, Fig. $1 B$ ). The third run was used as prestimulation baseline and compared with the poststimulation 2PD run. Thirty minutes of RSS led to an enhancement of tactile acuity, as expressed by a significant reduction of the 2PD threshold (post hoc difference pre-post $=0.24 \mathrm{~mm}, p<4.67 \times$ $10^{-6}$, paired Student's $t$ test, Fig. $1 B$ ), which is in line with previous studies using an identical protocol (Godde et al., 2000; Pleger et al., 2001, 2003; Dinse et al., 2003). Similar to earlier studies, the effect was specific for the stimulated right IF, while the discrimination threshold of the left control IF remained unchanged (difference prepost $=0.0058 \mathrm{~mm}, p<0.906$ ). Although RSS led to a significant improvement in discrimination abilities on average, individual learning success varied greatly, which corroborated previous findings using this procedure (Godde et al., 2000; Pleger et al., 2001; Hodzic et al., 2004). Separate analyses for the two different stimulation conditions yielded pre-post discrimination differences of $0.25 \mathrm{~mm}, p<$ $2.48 \times 10^{-5}$ and $0.20 \mathrm{~mm}, p<0.16$ for electrical and cutaneous stimulation respectively. Despite the fact that all subjects included in the study exhibited a significant EEG response to the RSS (see Materials and Methods), six (two vs four undergoing cutaneous vs electrical stimulation) of the 26 subjects showed no improvement after RSS. Notably the electrical stimulus intensity adjusted to $50 \%$ above the individual sensory threshold did not correlate with the degree of perceptual learning $(r=0.11$, $p<0.63)$.

\section{Central alpha rhythm power during resting-state before RSS predicts perceptual learning}

Next we analyzed how much the different ongoing neuronal states of the individual subjects, before and during RSS, might explain the observed differences in the learning success. In our analyses, all EEG channels and frequency bands ranging from delta to low gamma (i.e., $1-40 \mathrm{~Hz}$ ) were covered. Because we used tactile stimulation for RSS, we expected to find main effects for the oscillatory activity of sensorimotor cortex peaking in the alpha $(8-12 \mathrm{~Hz})$ and beta $(13-29 \mathrm{~Hz})$ band and referred to as rolandic mu and beta rhythms (Pfurtscheller and Lopes da Silva, 1999). We first correlated individual changes in discrimination with the channelwise spectral power of ongoing oscillatory EEG activity during the resting-state period before learning (henceforth referred to as resting-state power). Of all the analyzed frequency bands, we only found significant correlations in the alpha frequency band, where a single cluster of electrodes over the postcentral parietal cortex, contralateral to the stimulation site, showed a highly significant effect (cluster level $p<0.002$, maximum correlation in channel CP1: $r=0.60, p<0.0013$, Fig. 3, right column). Notably, this relation was specific to perceptual improvement, since it was not present for either the control left IF (corresponding channel CP2: $r=-0.06, p<0.77$, Fig. 3, left column), or for the perceptual performance before RSS (channel CP1: $r=0.095, p<0.64$ ). Thus, the individual ongoing resting-state central alpha rhythm power in the prelearning phase predicts the individual perceptual learning efficacy, and accounts for up to $36 \%$ of the overall variance in the perceptual learning rate (with a peak correlation coefficient of $r=0.6$, the maximum coefficient of determination $R^{2}$, or explained variance, is 0.36 ). Separate analyses for the two different stimulation conditions yielded for electrical stimulation a maximum correlation in channel C1: $r=0.61, p<0.0046$ but no significant correlation for the cutaneous group. 
Induced event-related central alpha rhythm change during RSS predicts perceptual learning

A well known feature of the central alpha rhythm is a decrease of amplitude during sensory or motor activity. This phenomenon has been termed ERD (Pfurtscheller and Lopes da Silva, 1999; Cheyne et al., 2003; Gaetz and Cheyne, 2006). To further analyze the impact of ongoing oscillations during the perceptual learning phase, we computed the induced change of oscillatory power after each train of stimulation, and correlated the individual's average power change with the perceptual learning outcome. As expected, the stimulation during RSS led to significant ERD of the central alpha rhythm in a large cluster of contralateral channels (cluster level $p<0.0005$ ). The strongest ERD was found in channel C3, located over the contralateral sensorimotor cortex $\left(-1.67 \mathrm{~dB}, p<1.64 \times 10^{-7}\right)$. Furthermore, in a single cluster of channels over the sensorimotor cortex contralateral to RSS the individual central alpha rhythm ERD was significantly correlated with the individual perceptual learning outcome (Fig. 4 left column, cluster level $p<0.0085$, maximum correlation in channel FC3: $r=-0.59, p<0.0015$, Pearson's linear correlation). The negative correlation coefficient indicates that higher learning success was associated with stronger power decrease. Again, the significant correlation was only present for the right IF exposed to RSS, but not for the left control IF (Fig. 4 right column, corresponding channel FC4: $r=0.046, p<0.82$ ). Thus, not only the ongoing resting-state power of the central alpha rhythm before learning, but also the central alpha rhythm response to the tactile stimuli delivered during RSS, was highly predictive of the perceptual improvement. The ERD strength in response to the RSS stimulation accounted for up to $34.8 \%$ of the overall variance in the perceptual learning rate $\left(R^{2}=0.348\right)$. We did not find a significant correlation in any of the other frequency bands, suggesting that the relationship is indeed specific to the central alpha rhythm. There was also no significant correlation between the electrical stimulus intensity set to $50 \%$ of the individual threshold and the degree of ERD (channel with maximum ERD: C3, $r=0.3$, $p<0.2$ ). Separate analyses for the two different stimulation conditions yielded for electrical stimulation a maximum correlation in channel FC3: $r=-0.56, p<0.01$ and for cutaneous stimulation a maximum correlation in channel CP3: $r=-0.83, p<$ 0.058. A correlation analysis between ERD and the peak-to-peak amplitude of the early component of the somatosensory evoked potential revealed for none of the EEG channels a significant correlation. Channel $\mathrm{C} 3$ that exhibited maximal ERD and ERP responses in the grand average yielded a correlation of $r=0.14 / p<0.50$.

EEG signatures of central alpha rhythm explain interindividual differences in perceptual learning We identified two EEG signatures of ongoing activity that predicted the individual perceptual learning efficacy, each acanalysis.

\section{Correlation: $\alpha$-band ERD vs. 2 PD change}

right stimulated IF

left control IF
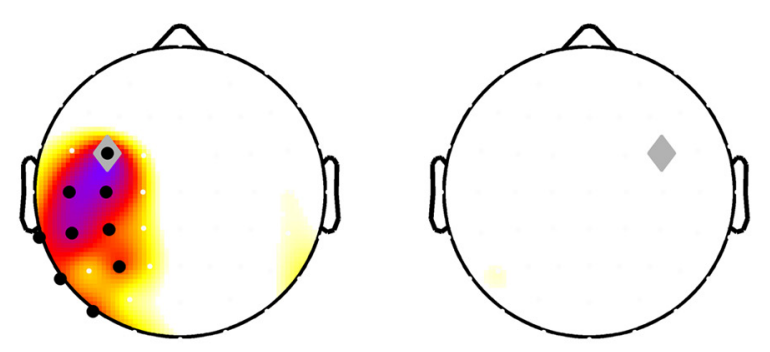

FC3: $R=-0.59 / P<0.0015$

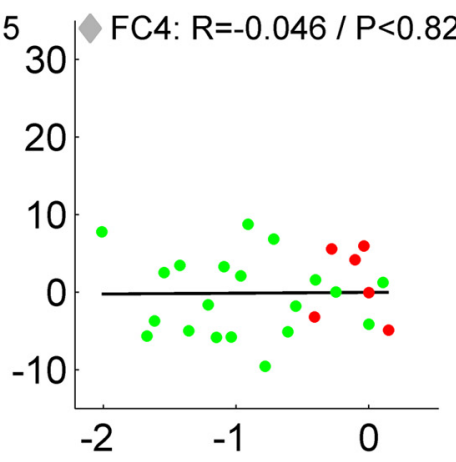

ERD $(d B)$

Figure 4. Correlation between 2PD change (left column, stimulated right IF; right column, control left IF) and alpha band ERD during RSS. A, Top row, Scalp distribution of Pearson's correlation coefficients $R$ and corresponding $p$-values. Black dots, Channels significant cluster $\left(p_{\text {clust }}<0.0025\right)$. Gray diamond, Channel FC3 with maximum correlation to learning rate (for right earners in green $(n=20)$, other subjects in red for illustration purposes - no categorization/collapsing was done for correlation

counting for $\sim 35 \%$ of the interindividual variance in perceptual improvement. The two predictor variables-resting-state central alpha rhythm power before stimulation (regressor I) and central alpha rhythm ERD during stimulation (regressor II) are known to exhibit a close relationship (Doppelmayr et al., 1998), and might be partially dependent. Therefore, the question arises as to how much of the behavioral variance is explained by both regressors together. In principle they could explain the same or independent parts of the variance in behavioral data- or a combination of both. One way to solve this problem is to create an orthogonal set of predictor variables (i.e., by orthogonalizing one regressor to the other). In such an orthogonal set, it is ensured that each predictor variable explains a unique part of the variance in the observed variable (in our case the perceptual learning rate). Figure 5 shows a topographic distribution of the total explained variance that is explained by the sum of the orthogonal set of the two predictor variables, obtained by a modified GramSchmidt projection. A center of gravity is visible over the contralateral sensorimotor cortex (maximum at channel FC3: $\left.R^{2}=0.638\right)$. Interestingly, the orthogonalized predictor variables contributed almost equally to the total explained variance (regressor I/II: $R^{2}=0.289 / 0.349$ ). These results, together with the different topographies seen in Figures 3 and 4, suggest that the two EEG signatures do reflect two distinct sources of the central alpha rhythm accounting for different aspects of the interindividual variability in perceptual improvement. In summary, our findings suggest that $\sim 64 \%$ of the interindi- 


\section{Explained Variance}

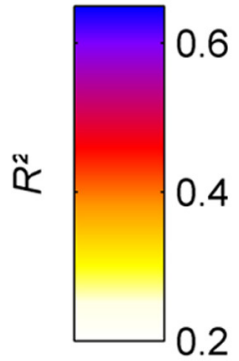

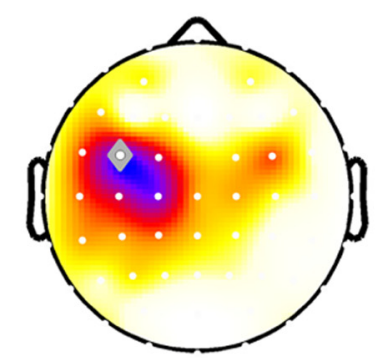

right stimulated IF

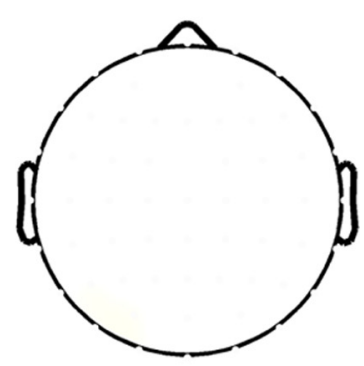

left control IF

Figure 5. Topographic scalp map showing the total variance of individual gain in tactile acuity (i.e., 2PD threshold change) after RSS explained by the (channelwise) orthogonalized set of EEG predictor variables, alpha band resting-state power and alpha band ERD (left column, right stimulated IF; right column, left control IF). The explained variances (i.e., the respective $R^{2}$ ) of both predictor variables were added to yield the total explained variance. The channel with the maximum explained variance is indicated as a gray diamond $\left(\mathrm{FC} 3, R^{2}=0.638\right)$.

vidual differences of perceptual improvement, induced by exposure-based perceptual learning, can be explained by ongoing oscillatory activity of the sensorimotor system before and during the learning phase.

\section{Discussion}

We aimed to identify neuronal determinants that explain interindividual differences in exposure-based perceptual learning through repetitive coactivation of the nerves of the fingers. This type of stimulation improves tactile discrimination abilities and enlarges cortical representation of the respective skin area (Godde et al., 2000; Pleger et al., 2001, 2003; Dinse et al., 2003). Due to the coactive nature of stimulation, the effect is suggested to reflect Hebbian learning. In other words, repeated simultaneous activation of many neural receptive fields leads to increased synaptic efficacies between neuronal representations. When using a modified version of the coactivation protocol consisting of a single, small stimulation site instead of one large area, no changes in perception or cortical maps occur (Pleger et al., 2003; Ragert et al., 2008). This implies that spatial summation requirements, indicative of cooperative processes, need to be fulfilled to drive behavioral change.

We found that individual perceptual improvement is predicted by two specific features of ongoing neuronal oscillations. The resting-state parietal alpha rhythm $(8-12 \mathrm{~Hz})$ power before perceptual learning and central alpha rhythm ERD during the learning phase accounted for $64 \%$ of the interindividual variance in perceptual learning.

Nonspecific factors, such as interindividual differences in attention and vigilance, appear unlikely explanations for this phenomenon for the following reasons: First, there was no relation between ongoing activity and interindividual fluctuations of tactile acuity for the unstimulated left IF, which was not treated with RSS and therefore served as a behavioral control. Conceivably, a global shift of attention or vigilance would affect the performance of both the stimulated and the unstimulated finger. Second, the behavioral baseline performance of the right stimulated IF before learning did not correlate with parameters of ongoing activity, either before or after learning - however, a vigilance/attention effect would require such a correlation. Third, the central alpha rhythm power that predicted the learning outcome showed a lateralized topography with a peak over the sensorimotor cortex contralateral to the RSS protocol. Typically, global vigilance and/or attention effects are related to more symmetrical changes in the fronto-occipital distribution and amplitude of the alpha rhythm. Finally, the direction of the observed relation between central alpha rhythm power and performance argues against a global vigilance/attention effect: In our data, high values of resting-state central alpha rhythm power were linked to higher perceptual learning efficacy, whereas high attention or vigilance would be indicated by low alpha-rhythm power (Thut et al., 2006; Mazaheri et al., 2009).

It is assumed that incoming stimuli and internal ongoing neuronal dynamics interact (Buonomano and Maass, 2009). Supposedly not only the firing rates at the time of stimulus arrival matter-but also "hidden" neuronal states, such as altered synaptic efficiencies (Buonomano et al., 2009). Indeed, a close relationship between stimulus-evoked activity and spontaneously ongoing oscillations have been reported not only for EEG/MEG (Becker et al., 2008a, 2011; Mazaheri and Jensen, 2008), but also in invasive animal recordings (Arieli et al., 1996) and fMRI (Fox et al., 2007).

We provide further evidence for this close relationship by showing a positive relation between resting-state alpha rhythm power before perceptual learning, and the increase in behavioral performance due to the learning process. These findings are in contrast to other data indicating negative relations between ongoing (prestimulus) alpha power and evoked activity/perception in somatosensory (Linkenkaer-Hansen et al., 2004) and visual systems (Thut et al., 2006; Becker et al., 2011), and also contradict results demonstrating decreased excitability of cortex regions expressing higher alpha power (Romei et al., 2008). Part of these differences might be due to the fact that we examined interindividual variability of resting alpha-rhythm power before learning and not intraindividual trial-by-trial fluctuations of prestimulus central alpha-rhythm power. The prevailing theory postulates that increased alpha activity reflects top-down inhibitory control of task-irrelevant brain resources (Pfurtscheller and Lopes da Silva, 1999; Klimesch et al., 2007) known as "gating by inhibition" (Jensen and Mazaheri, 2010). Indeed, top-down processes modulate alpha activity (lateralization) in sensorimotor regions presumably reflecting frontally controlled disengagement of task-irrelevant regions (Haegens et al., 2011).

In our study though high alpha-rhythm power may indicate that learning-relevant regions are not engaged in any process and hence exhibits an idling state with high reactivity and preparedness to process incoming learning stimuli. This interpretation is in line with a theory about the possible functional meaning of the brain's intrinsic activity, proposing that intrinsic activity could serve the purpose of facilitating responses to stimuli by increasing the gain of neurons through a constant seesaw between excitation and inhibition (Haider et al., 2006; Raichle, 2006). Positive relations between alpha power and fMRI signals have been reported for the default mode network (DMN), a network of coherent intrinsic activity during the resting state (Mantini et al., 2007). That high alpha states in parietal cortex are relevant for learning, suggests functional relevance of the DMN state before learning- 
similar to its role for verbal working memory (Hampson et al., 2006).

While the link between alpha rhythm activity and perceptual learning —as revealed in the present study—is new, there exist studies that report a relation between the alpha rhythm and visual short-term memory performance. For such tasks a difference in alpha frequency has been revealed for good and bad performers (Klimesch et al., 1990, 1993) and visual memory capacity correlated with alpha amplitude ipsilateral to relevant items supposedly reflecting suppression of irrelevant information (Sauseng et al., 2009).

It needs to be noted that the topographies of the variables relevant for learning- i.e., baseline alpha and alpha ERD-do differ (Figs. 3, 4)-despite the fact that they are both located over central brain areas and hence are referred to here to as "central alpha." While for the baseline alpha rhythm we find a posteroparietal distribution, the topography of alpha ERD is located over sensorimotor cortex. For the posteroparietal alpha rhythm we have shown previously a link to the DMN in fMRI as explicated above (Becker et al., 2008b; Ritter et al., 2008). The sensorimotor alpha rhythm is also known as rolandic alpha or mu-rhythm (Pfurtscheller and Lopes da Silva, 1999).

As to the positive correlation between the central alpha (or $\mathrm{mu}$ ) rhythm ERD during perceptual learning, and behavioral improvement, our data support the notion that learning-relevant brain regions are more activated in "good learners." The ERD of the central alpha rhythm in response to incoming stimuli (Cheyne et al., 2003; Gaetz et al., 2006) has been interpreted as a marker for cortical activation (Pfurtscheller, 2006).

Other studies have shown that the amount of perceptual improvement after exposure-based perceptual learning is positively correlated to the amount of local cortical reorganization as indicated by mapping of somatosensory evoked potentials (Pleger et al., 2001; Dinse et al., 2003), evoked fMRI responses (Hodzic et al., 2004) or changes of cortical excitability (Höffken et al., 2007). Our data extend these studies by demonstrating that also cortical reorganization is related to the amount of central alpha rhythm ERD. The fact that we see a desynchronization of the scalp alpha rhythm during the perceptual learning phase does not necessarily exclude an active mechanistic role of these oscillations at the cellular level. Both cessation and amplitude decreases of oscillations on the one hand and desynchronized but persisting oscillatory activity on the other hand can lead to the same attenuation of large scale oscillations (Telenczuk et al., 2010).

Our study highlights the relevance of ongoing brain states by demonstrating that the interindividual variability of the learning success is explained to a large extent by resting parietal alpha rhythm before, and central alpha-more specifically murhythm ERD during perceptual learning. This observation is in line with earlier findings that point to a significant relationship between resting-state network activity and perceptual learning. A recent study showed that interindividual variability in perceptual performance is predicted by functional connectivity patterns of large-scale resting-state networks before learning (Baldassarre et al., 2012). Another study revealed that individual performance changes after perceptual learning are predicted by alterations of resting-state functional connectivity (Lewis et al., 2009). Our findings are also consistent with the work of Sigman et al. (Sigman et al., 2005) who showed that DMN activity predicted perceptual learning.

It remains to be clarified whether differences observed in alpha rhythm power before learning reflect a spontaneous expression of individual brain states or a fingerprint-like property. In the latter case, differences in central alpha rhythm power might be coupled to gross anatomical or genetic differences.

In conclusion, our results show that a person's ability to learn is dependent on the brain state before and during perceptual learning. This provides a fundamental contribution to the puzzle of the origins of intersubject variability in perceptual learning. Conceivably, better understanding of the conditions that facilitate different types of learning is instrumental for further development of strategies in the clinical and educational realms. Concretely, one apparent consequence of our results is the possibility to manipulate individual alpha power through neurofeedback to enhance perceptual learning.

\section{References}

Arieli A, Sterkin A, Grinvald A, Aertsen A (1996) Dynamics of ongoing activity: explanation of the large variability in evoked cortical responses. Science 273:1868-1871. CrossRef Medline

Baldassarre A, Lewis CM, Committeri G, Snyder AZ, Romani GL, Corbetta M (2012) Individual variability in functional connectivity predicts performance of a perceptual task. Proc Natl Acad Sci U S A 109:3516-3521. CrossRef Medline

Becker R, Ritter P, Villringer A (2008a) Influence of ongoing alpha rhythm on the visual evoked potential. Neuroimage 39:707-716. CrossRef Medline

Becker R, Ritter P, Villringer A (2008b) Visual System. In EEG-fMRI: physiological basis, technique, and applications (Mulert C, Lemieux L, eds). Berlin: Springer.

Becker R, Reinacher M, Freyer F, Villringer A, Ritter P (2011) How ongoing neuronal oscillations account for evoked FMRI variability. J Neurosci 31:11016-11027. CrossRef Medline

Beste C, Wascher E, Güntürkün O, Dinse HR (2011) Improvement and impairment of visually guided behavior through LTP- and LTD-like exposure-based visual learning. Curr Biol 21:876-882. CrossRef Medline

Bell AJ, Sejnowski TJ (1995) An information-maximization approach to blind separation and blind deconvolution. Neural Comput 7:1129-1159. Medline

Boly M, Balteau E, Schnakers C, Degueldre C, Moonen G, Luxen A, Phillips C, Peigneux P, Maquet P, Laureys S (2007) Baseline brain activity fluctuations predict somatosensory perception in humans. Proc Natl Acad Sci U S A 104:12187-12192. CrossRef Medline

Bullmore ET, Suckling J, Overmeyer S, Rabe-Hesketh S, Taylor E, Brammer MJ (1999) Global, voxel, and cluster tests, by theory and permutation, for a difference between two groups of structural MR images of the brain. IEEE Trans Med Imaging 18:32-42. CrossRef Medline

Buonomano DV, Maass W (2009) State-dependent computations: spatiotemporal processing in cortical networks. Nat Rev Neurosci 10: 113-125. CrossRef Medline

Buonomano DV, Bramen J, Khodadadifar M (2009) Influence of the interstimulus interval on temporal processing and learning: testing the state-dependent network model. Philos Trans R Soc Lond B Biol Sci 364:1865-1873. CrossRef Medline

Cheyne D, Gaetz W, Garnero L, Lachaux JP, Ducorps A, Schwartz D, Varela FJ (2003) Neuromagnetic imaging of cortical oscillations accompanying tactile stimulation. Cogn Brain Res 17:599-611. CrossRef

Crist RE, Li W, Gilbert CD (2001) Learning to see: experience and attention in primary visual cortex. Nat Neurosci 4:519-525. Medline

Deco G, Jirsa VK, McIntosh AR (2011) Emerging concepts for the dynamical organization of resting-state activity in the brain. Nat Rev Neurosci 12:43-56. CrossRef Medline

Delorme A, Makeig S (2004) EEGLAB: an open source toolbox for analysis of single-trial EEG dynamics including independent component analysis. J Neurosci Methods 134:9-21. CrossRef Medline

Dinse HR, Ragert P, Pleger B, Schwenkreis P, Tegenthoff M (2003) Pharmacological modulation of perceptual learning and associated cortical reorganization. Science 301:91-94. CrossRef Medline

Dinse HR, Kleibel N, Kalisch T, Ragert P, Wilimzig C, Tegenthoff M (2006) Tactile coactivation resets age-related decline of human tactile discrimination. Ann Neurol 60:88-94. CrossRef Medline

Doppelmayr MM, Klimesch W, Pachinger T, Ripper B (1998) The functional significance of absolute power with respect to event-related desynchronization. Brain Topogr 11:133-140. CrossRef Medline 
Fahle M, Henke-Fahle S (1996) Interobserver variance in perceptual performance and learning. Invest Ophthalmol Vis Sci 37:869-877. Medline

Fahle M, Edelman S, Poggio T (1995) Fast perceptual learning in hyperacuity. Vision Res 35:3003-3013. CrossRef Medline

Fahle M, Poggio T (2002) Perceptual learning. Cambridge: MIT.

Fox MD, Snyder AZ, Vincent JL, Raichle ME (2007) Intrinsic fluctuations within cortical systems account for intertrial variability in human behavior. Neuron 56:171-184. CrossRef Medline

Gaetz W, Cheyne D (2006) Localization of sensorimotor cortical rhythms induced by tactile stimulation using spatially filtered MEG. Neuroimage 30:899-908. CrossRef Medline

Godde B, Stauffenberg B, Spengler F, Dinse HR (2000) Tactile coactivationinduced changes in spatial discrimination performance. J Neurosci 20: 1597-1604. Medline

Haegens S, Händel BF, Jensen O (2011) Top-down controlled alpha band activity in somatosensory areas determines behavioral performance in a discrimination task. J Neurosci 31:5197-5204. CrossRef Medline

Haider B, Duque A, Hasenstaub AR, McCormick DA (2006) Neocortical network activity in vivo is generated through a dynamic balance of excitation and inhibition. J Neurosci 26:4535-4545. CrossRef Medline

Hampson M, Driesen NR, Skudlarski P, Gore JC, Constable RT (2006) Brain connectivity related to working memory performance. J Neurosci 26:13338-13343. CrossRef Medline

Hesselmann G, Kell CA, Eger E, Kleinschmidt A (2008) Spontaneous local variations in ongoing neural activity bias perceptual decisions. Proc Natl Acad Sci U S A 105:10984-10989. CrossRef Medline

Hodzic A, Veit R, Karim AA, Erb M, Godde B (2004) Improvement and decline in tactile discrimination behavior after cortical plasticity induced by passive tactile coactivation. J Neurosci 24:442-446. CrossRef Medline

Höffken O, Veit M, Knossalla F, Lissek S, Bliem B, Ragert P, Dinse HR, Tegenthoff M (2007) Sustained increase of somatosensory cortex excitability by tactile coactivation studied by paired median nerve stimulation in humans correlates with perceptual gain. J Physiol 584:463-471. CrossRef Medline

Jensen O, Mazaheri A (2010) Shaping functional architecture by oscillatory alpha activity: gating by inhibition. Front Hum Neurosci 4:186. Medline

Klimesch W, Schimke H, Ladurner G, Pfurtscheller G (1990) Alpha frequency and memory performance. J Psychophysiol 4:381-390.

Klimesch W, Schimke H, Pfurtscheller G (1993) Alpha frequency, cognitive load and memory performance. Brain Topogr 5:241-251. CrossRef Medline

Klimesch W, Sauseng P, Hanslmayr S (2007) EEG alpha oscillations: the inhibition-timing hypothesis. Brain Res Rev 53:63-88. CrossRef Medline

Lewis CM, Baldassarre A, Committeri G, Romani GL, Corbetta M (2009) Learning sculpts the spontaneous activity of the resting human brain. Proc Natl Acad Sci U S A 106:17558-17563. CrossRef Medline

Linkenkaer-Hansen K, Nikulin VV, Palva S, Ilmoniemi RJ, Palva JM (2004) Prestimulus oscillations enhance psychophysical performance in humans. J Neurosci 24:10186-10190. CrossRef Medline

Mantini D, Perrucci MG, Del Gratta C, Romani GL, Corbetta M (2007) Electrophysiological signatures of resting state networks in the human brain. Proc Natl Acad Sci U S A 104:13170-13175. CrossRef Medline

Maris E, Oostenveld R (2007) Nonparametric statistical testing of EEG- and MEG-data. J Neurosci Methods 164:177-190. CrossRef Medline

Mazaheri A, Jensen O (2008) Asymmetric amplitude modulations of brain oscillations generate slow evoked responses. J Neurosci 28:7781-7787. CrossRef Medline

Mazaheri A, Nieuwenhuis IL, van Dijk H, Jensen O (2009) Prestimulus alpha and mu activity predicts failure to inhibit motor responses. Hum Brain Mapp 30:1791-1800. CrossRef Medline

Pfurtscheller G (2006) The cortical activation model (CAM). Prog Brain Res 159:19-27. CrossRef Medline

Pfurtscheller G, Lopes da Silva FH (1999) Event-related EEG/MEG synchronization and desynchronization: basic principles. Clin Neurophysiol 110:1842-1857. CrossRef Medline

Pleger B, Dinse HR, Ragert P, Schwenkreis P, Malin JP, Tegenthoff M (2001) Shifts in cortical representations predict human discrimination improvement. Proc Natl Acad Sci U S A 98:12255-12260. CrossRef Medline

Pleger B, Foerster AF, Ragert P, Dinse HR, Schwenkreis P, Malin JP, Nicolas V, Tegenthoff M (2003) Functional imaging of perceptual learning in human primary and secondary somatosensory cortex. Neuron 40:643-653. CrossRef Medline

Ragert P, Kalisch T, Bliem B, Franzkowiak S, Dinse HR (2008) Differential effects of tactile high- and low-frequency stimulation on tactile discrimination in human subjects. BMC Neurosci 9:9. CrossRef Medline

Raichle ME (2006) Neuroscience. The brain's dark energy. Science 314: 1249-1250. CrossRef Medline

Ritter P, Greicius MD, Becker R, Vilringer A (2008) Relation between spatially and spectrally confined EEG rhythms and fMRI resting state networks. In 14th Annual Meeting of the OHBM, Melbourne, Australia, June $15-19$.

Romei V, Brodbeck V, Michel C, Amedi A, Pascual-Leone A, Thut G (2008) Spontaneous fluctuations in posterior alpha-band EEG activity reflect variability in excitability of human visual areas. Cereb Cortex 18:2010-2018. Medline

Sasaki Y, Nanez JE, Watanabe T (2010) Advances in visual perceptual learning and plasticity. Nat Rev Neurosci 11:53-60. CrossRef Medline

Sauseng P, Klimesch W, Heise KF, Gruber WR, Holz E, Karim AA, Glennon M, Gerloff C, Birbaumer N, Hummel FC (2009) Brain oscillatory substrates of visual short-term memory capacity. Curr Biol 19:1846-1852. CrossRef Medline

Schubert R, Haufe S, Blankenburg F, Villringer A, Curio G (2009) Now you'll feel it, now you won't: EEG rhythms predict the effectiveness of perceptual masking. J Cogn Neurosci 21:2407-2419. CrossRef Medline

Seitz AR, Dinse HR (2007) A common framework for perceptual learning. Curr Opin Neurobiol 17:148-153. CrossRef Medline

Sigman M, Pan H, Yang Y, Stern E, Silbersweig D, Gilbert CD (2005) Topdown reorganization of activity in the visual pathway after learning a shape identification task. Neuron 46:823-835. CrossRef Medline

Telenczuk B, Nikulin VV, Curio G (2010) Role of neuronal synchrony in the generation of evoked EEG/MEG responses. J Neurophysiol 104: 3557-3567. CrossRef Medline

Thut G, Nietzel A, Brandt SA, Pascual-Leone A (2006) Alpha-band electroencephalographic activity over occipital cortex indexes visuospatial attention bias and predicts visual target detection. J Neurosci 26:9494-9502. CrossRef Medline

Watanabe T, Náñez JE, Sasaki Y (2001) Perceptual learning without perception. Nature 413:844-848. CrossRef Medline

Wichmann FA, Hill NJ (2001) The psychometric function. I. Fitting, sampling, and goodness of fit. Percept Psychophys 63:1293-1313. Medline 